\title{
The limb-darkening for spherically symmetric NextGen model atmospheres: A-G main-sequence and sub-giant stars ${ }^{\star}$
}

\author{
A. Claret ${ }^{1}$ and P. H. Hauschildt ${ }^{2}$ \\ 1 Instituto de Astrofísica de Andalucía, CSIC, Apartado 3004, 18080 Granada, Spain \\ 2 Hamburger Sternwarte, Gojenbergsweg 112, 21029 Hamburg, Germany \\ e-mail: Peter.Hauschildt@hs.uni-hamburg.de
}

Received 4 June 2003 / Accepted 29 August 2003

\begin{abstract}
We compute passband-integrated specific intensities for spherically symmetric models generated with the PHOENIX code. The models cover the range $5000 \mathrm{~K} \leq T_{\text {eff }} \leq 10000 \mathrm{~K}$ with surface gravities between 3.5 and 5.5 , in steps of 0.5 . The adopted mixing-length parameter is 2.0 pressure scale heights, while the microturbulent velocity is $2.0 \mathrm{~km} \mathrm{~s}^{-1}$. The calculations were carried out for four photometric systems: Strömgren, Johnson, Geneva and Walraven, completing 24 filters. The series introduced by Claret (2000) and a new bi-parametric (exponential) law are shown to be able to describe the intensity distribution of spherical models with acceptable $\sigma$. Simple limb-darkening laws do not produce accurate fits to the computed radiation fields. On the other hand, the concept of "quasi" spherical models is introduced and we present the corresponding limb-darkening coefficients for the most commonly adopted limb-darkening laws for the same filters.
\end{abstract}

Key words. stars: atmospheres - stars: binaries: eclipsing

\section{Introduction}

The limb-darkening coefficients (LDC) are a basic tool in several fields of Astrophysics such as double-lined eclipsing binaries (the main source of stellar masses), measurement of stellar diameters, line profiles of rotating stars and more recently the transit of extra-solar planets, gravitational microlensing and optical interferometry.

The current database of stellar atmosphere models is large, compared to those available only a few years ago (see for example the CDROMs by Kurucz 1993 or the grids published by the PHOENIX and MARCS groups). Most of these models were computed under the assumption of plane-parallel symmetry and there are only a few investigations of the effects of sphericity on the intensity distribution, e.g. Orosz \& Hauschildt (2000). However, no detailed information of the limb-darkening was given since that the main goal of the authors was to present a new light curve synthesis code rather than to provide tables of the LDC.

The most commonly adopted limb-darkening laws are linear, quadratic, square-root and logarithmic, respectively:

$\frac{I(\mu)}{I(1)}=1-u(1-\mu)$

\footnotetext{
Send offprint requests to: A. Claret, e-mail: claret@iaa.es

* Tables 2-17 are only available in electronic form at http://www . edpsciences.org
}

$\frac{I(\mu)}{I(1)}=1-a(1-\mu)-b(1-\mu)^{2}$

$\frac{I(\mu)}{I(1)}=1-c(1-\mu)-d(1-\sqrt{\mu})$

$\frac{I(\mu)}{I(1)}=1-e(1-\mu)-f \mu \ln (\mu)$

where $I(1)$ is the specific intensity at the center of the disk, $u, a, b, c, d, e, f$ are the respective LDC. Claret (2000) introduced a non-linear law capable of reproducing the intensities of plane-parallel models very well. This law can be written as:

$$
\frac{I(\mu)}{I(1)}=1-\sum_{k=1}^{4} a_{k}\left(1-\mu^{\frac{k}{2}}\right)
$$

Two numerical methods are often used to generate the LDC: the Least-Square (LSM) and the Flux Conservation (FCM). Both numerical procedures have been the subject of extensive discussions so we do not repeat the argumentation here. The main conclusion is that LSM is the most consistent approach (see Claret 2000 for more information). Therefore, we adopt here the LSM in all LDC calculations. The corresponding $\sigma \mathrm{s}$ are given by

$\sigma=\frac{1}{N-M}\left(\sum_{i=1}^{N}\left(y_{i}-Y_{i}\right)^{2}\right)^{1 / 2}$ 
where $y_{i}$ is the model intensity at the point $i, Y_{i}$ the fitted function at the same point; $N$ is the number of points and $M$ is the number of coefficients to be adjusted.

In the present paper we investigate the specific intensities of spherical models generated with the PHOENIX code for effective temperatures between 5000 and $10000 \mathrm{~K}$, with $\log g$ varying between 3.5 and 5.5, appropriate for A-G spectral types. In the first part of this paper we briefly describe the properties of the spherical models and present the actual integrated specific intensities in four photometric systems (24 filters): Strömgren, Johnson, Walraven and Geneva. Next, the concept of a "quasi" spherical model is introduced and the respective LDC are computed for the most commonly used limb-darkening approximations for the above photometric systems.

\section{A brief description of the PHOENIX models and the actual integrated specific intensities}

The present models were computed using the current version (13) of the PHOENIX code and the most recent setup of the microphysics. The models are characterized by the effective temperature, $\log g$ and mass. We adopted for the present calculations a mass of $1 M_{\odot}$. The selection of the mass has a minor influence on the results provided that it is not changed by large factors. The stellar radius $R$ is set by the condition $g_{\text {grav }}=G M / R^{2}$, where we define $g_{\text {grav }}$ as the gravitational acceleration at $\tau_{\text {std }}=1, G$ is the gravitational constant, and $\tau_{\text {std }}$ is the optical depth in the continuum at $1.2 \mu$. The luminosity $L$ of the model is then given by $L=4 \pi R^{2} \sigma T_{\mathrm{eff}}^{4}$. For convenience, our model grid is based on the set of parameters $\left(T_{\mathrm{eff}}, \log g\right.$, $M / H)$. The above formulae and the structures of the model atmospheres can be used to transform them to any target set of parameters (e.g., for a different definition of $\tau_{\text {std }}$ ). The range of effective temperatures is $5000 \mathrm{~K} \leq T_{\text {eff }} \leq 10000 \mathrm{~K}$ and the values of $\log g$ are between 3.5 and 5.5. The adopted microturbulent velocity was $2.0 \mathrm{~km} \mathrm{~s}^{-1}$ and the mixing-length parameter was 2.0, based on calibrations of $\mathrm{M}$ type stars at $T_{\text {eff }} \approx$ 2800 K. 50 radial points were considered for each model and the specific intensities were computed for $99 \mu$ points with a distribution that is adapted to each atmospheric structure. About 53000 wavelengths were considered ranging from 10 to $9 \times 10^{6} \AA$ with steps of $2-50 \AA$. Several million atomic and molecular lines were taken into account, the exact number depends on the parameters of the model in question. All this information requires around $130 \mathrm{MB}$ per model radiation field spectrum. For $\mathrm{F}$ and $\mathrm{G}$ stars, the presence of temperature minima and chromospheres influence the drop-off region. However, it will only affect wavelengths that are optically thick in the chromospheric regions (or where there is substantial emission from the chromosphere). These regions are not included in the models and we do not expect that, for the colors that are considered in this paper, they make a substantial difference. For line core of lines like $\mathrm{Ca}$ II $\mathrm{H}+\mathrm{K}$ this chromosphere certainly will have to be considered. More detailed information on the numerical methods and input physics can be found in Hauschildt et al. (1999), Hauschildt et al. (1999) and Hauschildt \& Baron (1999).
In order to compute the specific intensities for a given passband we perform the convolution of the terrestrial atmospheric transmission, the filters transmission, the detector sensitivity and the double reflection of a aluminium mirror, represented by the function $S(\lambda)$ following the equation:

$I_{a}(\mu)=\frac{\int_{\lambda_{1}}^{\lambda_{2}} I(\lambda, \mu) S(\lambda) \mathrm{d} \lambda}{\int_{\lambda_{1}}^{\lambda_{2}} S(\lambda) \mathrm{d} \lambda}$

where $I(\lambda, \mu)$ is the monochromatic specific intensity (for details see Claret 2000).

There is a notorious difference between the normalized specific intensities computed using the plane-parallel and spherical geometry. In the plane-parallel approximation the specific intensity near the border of the disk is finite, while in spherically symmetrical models this quantity is very small, almost zero. There is a sudden drop off which depends on the effective temperature, $\log g$ and the passband (see Figs. 1, 2). These drop offs are caused by the decreased matter-radiation interaction near the limb of the star. Such behavior seems to indicate that simple limb-darkening laws are not capable of describing the distribution of the specific intensities with sufficient accuracy. Moreover, it is expected that the influence of sphericity effects will be not negligible. The most immediate effect is "lost light" since plane-parallel models predict a less pronounced decline at the border of the disk (Orosz \& Hauschildt 2000).

As explained by Claret (2000) and Orosz \& Hauschildt (2000) it is important to extract the maximum information possible from the stellar atmosphere models concerning limb-darkening. We make the actual specific intensities available (upon request) for four photometric systems: Strömgren, Johnson, Geneva and Walraven, 24 filters total. In order to differentiate the Walraven and Geneva filters from those by Johnson we denote the former as $G U G B 1 G B G B 2 G V 1 G V G G W W W U W L W B W V$. Figures 1, 2 show the behavior of the specific intensities for two models with $T_{\text {eff }}=10000 \mathrm{~K}$ and $5000 \mathrm{~K}$, both with $\log g=4.0$. Hereafter, we denote a particular model as $\left[T_{\text {eff }}\right.$, $\log g$ ], for the sake of clarity. In addition to the general dropoff near the limb, we can see in Figs. 1, 2 that these are more pronounced for larger effective wavelengths (filters $R I J H K$ ). Another characteristic in both figures is the steeper dependency of the integrated specific intensities in models with lower effective temperature. Note that before the drop-off point is reached, a model shows an almost linear behavior, as expected even for plane-parallel approximations in this effective temperature and gravity range.

Of course, the use of the actual integrated specific intensities is better than to adopt a given limb-darkening law resulting from a numerical adjustment. On the other hand, the use of LDC delivers a faster and more economic method for many applications. Due to the special characteristics of the intensity distributions produced by spherical models, simple laws are not capable of accurate fits, as shown by Orosz \& Hauschildt (2000) and confirmed by numerical experiments performed here. Only two laws (Eqs. (5) and (8)) were able to describe the center to limb variation with an acceptable accuracy in the case of spherical models. 

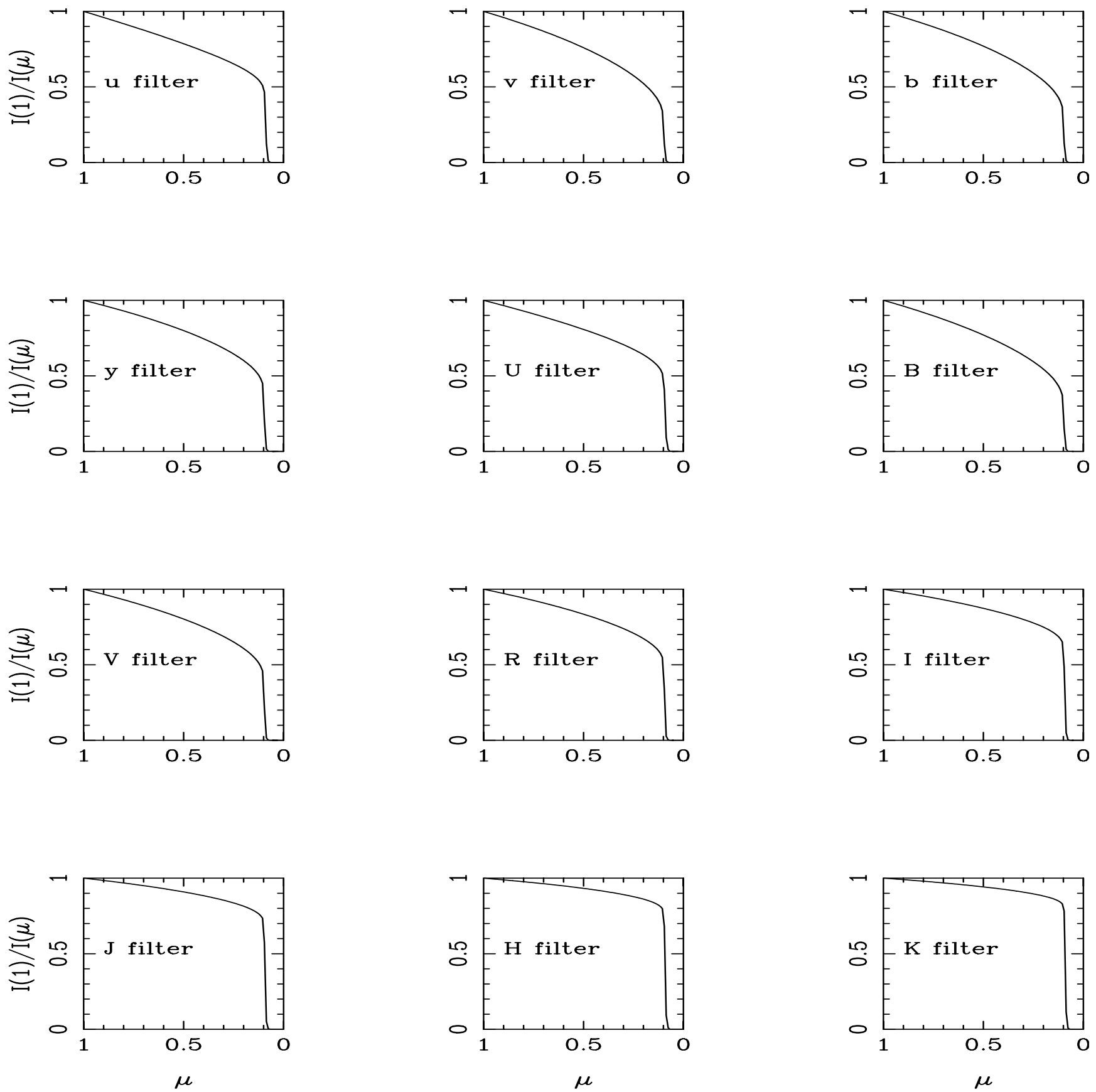

Fig. 1. The actual specific intensity distribution for a $[10000,4.0]$ model. Results for the Strömgren and Johnson systems.

As mentioned in the previous paragraph, we introduce a new bi-parametric approximation which can be written as:

$\frac{I(\mu)}{I(1)}=1-g(1-\mu)-\frac{h}{\left(1-e^{\mu}\right)}$.

Figures 3 and 4 illustrate the capability of Eqs. (5) and (8) to represent the intensities as a function of the emergent angle $\mu$ for a $[5000,4.0]$ model. Both approximations are very good at shorter effective wavelengths including the drop-off region but for larger effective wavelengths the fits are not as good, although they are still acceptable. In general, Eq. (8) produces the smallest $\sigma \mathrm{s}$.

\section{The "quasi" spherical models}

If one considers only the $\mu$ points previous to the drop-offs, the shape of the curves is similar to those with the same $T_{\text {eff }}$ and $\log g$ but computed with plane-parallel geometry (Fig. 5). Therefore, we define a "quasi" spherical model as one computed using spherical symmetry but LDC computed without considering the points within the drop-off region. Such a concept is very useful, for example, in circumstances where the effects of sphericity are not important. The two models represented in Fig. 5 were computed with the same code but under very different input physics:the number of lines taken 

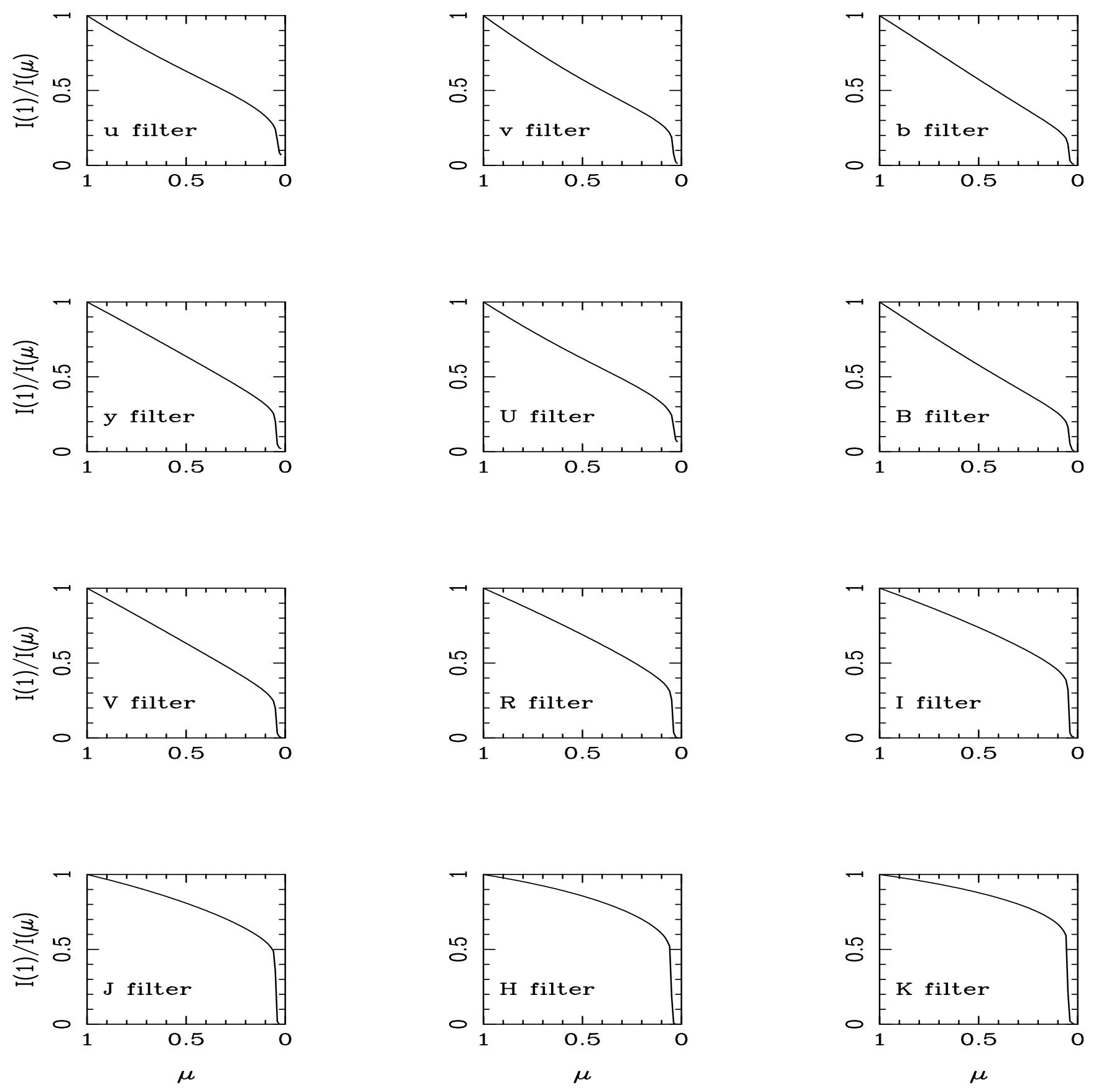

Fig. 2. The same as in Fig. 1 but for a $[5000,4.0]$ model.

into account, the number of $\mu$ points, distribution and number of $\lambda$ points, different adopted mixing-length parameters and, mainly, the adopted geometry. Even taking into account all these differences the distribution of intensities are not so different. The differences may be even smaller than those shown in Fig. 5 if the plane-parallel model is computed with the same input physics (see Fig. 6).

We have performed LDC calculations for the 24 filters applying the linear, quadratic, root-square, logarithmic, exponential laws and the approach given in Eq. (8). In Fig. 6 we show the results for a $[7000,4.0]$ model for the Walraven and Geneva filters using Eq. (5). The fits are very good for any passband. In fact, the dashed lines which denote the fits are indistinguishable from the asterisks which represent the actual integratedintensities. For each panel in Fig. 6 we plotted (full lines) the integrated-intensities for a plane-parallel model with the same effective temperature and surface gravity. By inspecting such Fig. 6 we can conclude that the the concept of "quasi" spherical models is fully supported when the microphysics input are similar. 

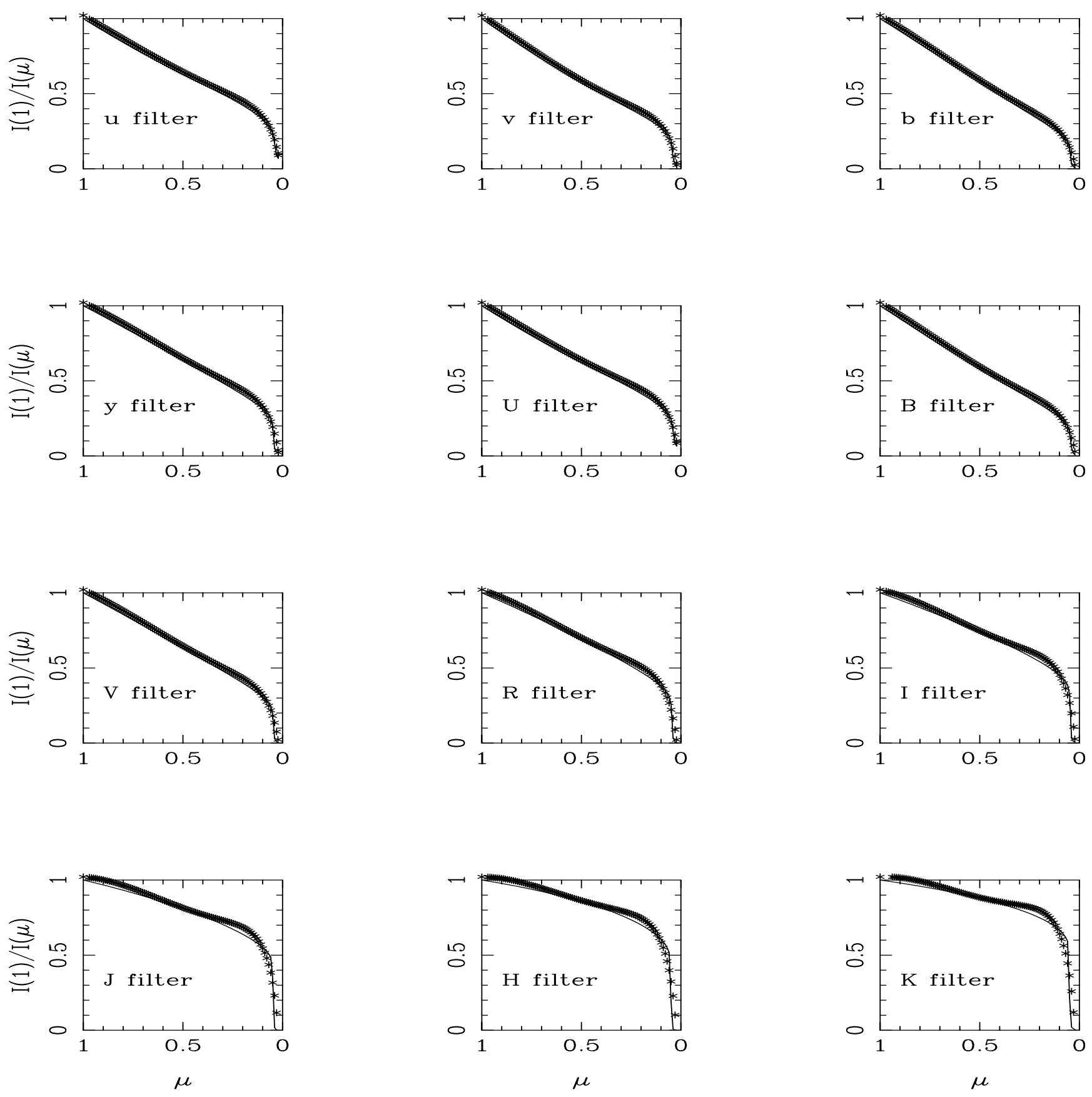

Fig. 3. The same as in Fig. 2. The asterisks indicate the fitting by applying Eq. (5).

Table 1 summarizes the results, which can be retrieved directly by anonymous ftp.

\section{Summary and conclusions}

We have computed integrated specific intensities for 24 passbands using recent spherical models generated by the PHOENIX code with effective temperatures between $5000 \mathrm{~K}$ and $10000 \mathrm{~K}$ and $\log g$ between 3.5 and 5.5. Only in two cases (the series proposed by Claret 2000 and an exponential law) was it possible to fit spherical models within an acceptable accuracy. On the other hand, we have introduced the concept of "quasi" spherical models, considering only the points previous to the drop-offs. The LDC for these models were computed using the most common limb-darkening laws. These "quasi" spherical models are also useful since they cover the gap in effective temperature indicated by Claret (1998).

Acknowledgements. The Spanish DGYCIT (PB98-0499) is gratefully acknowledged for its support during the development of this work. 

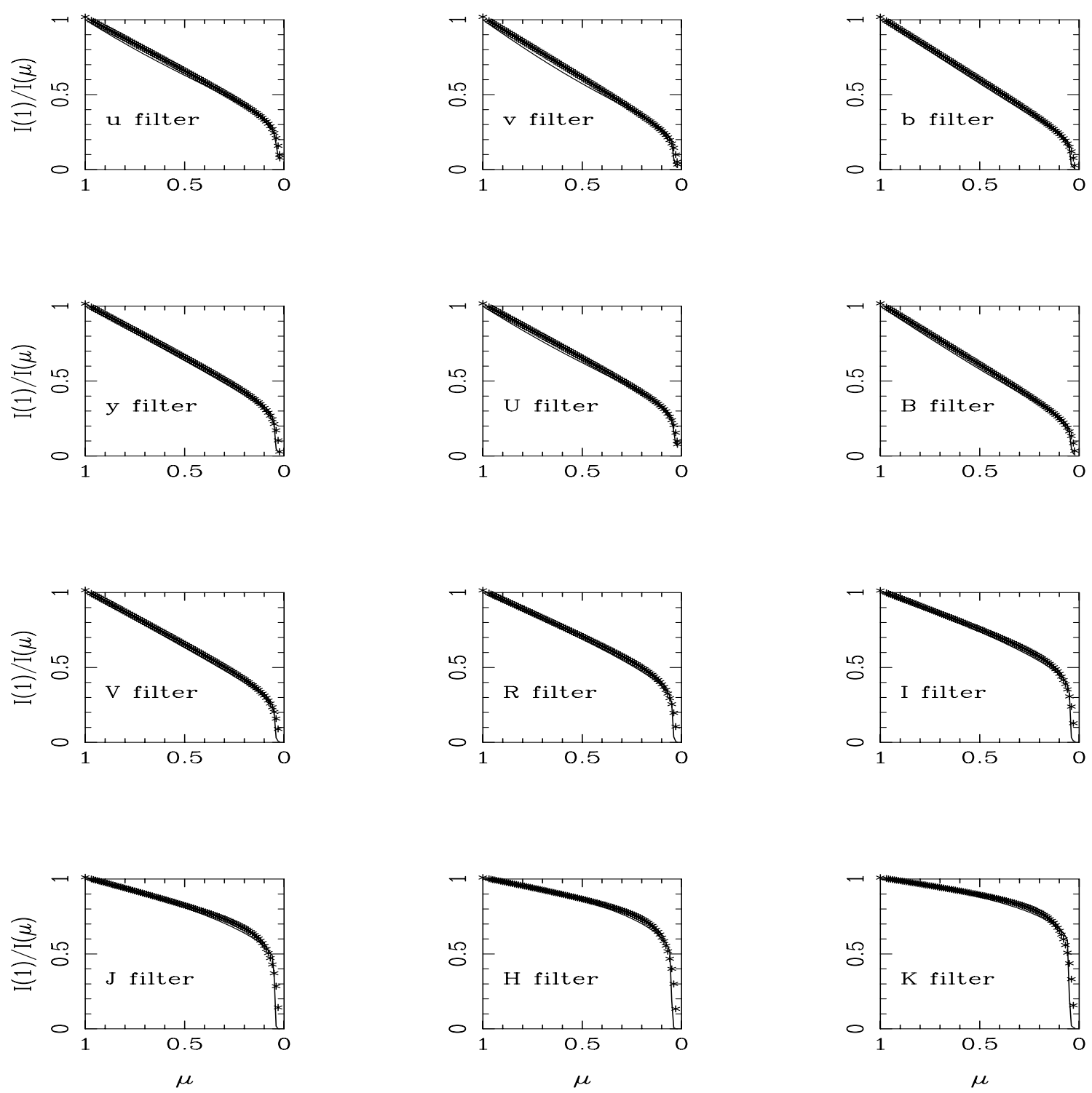

Fig. 4. The same as in Fig. 2. The asterisks indicate the fitting by applying Eq. (8).

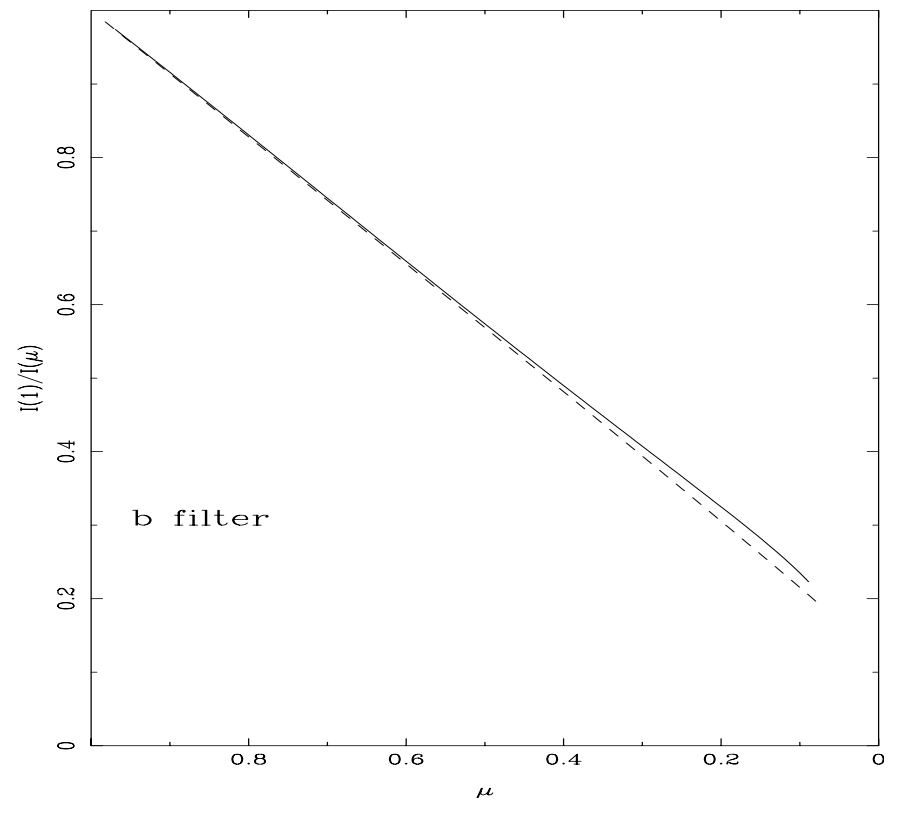

Fig. 5. NextGen model $[5000,4.0]$ following spherical symmetry (continuous line, present work) and the same model but computed under the plane-parallel approach (dashed line, see Claret 1998 for a description of the PHOENIX code). 
Table 1. Integrated intensities and limb-darkening coefficients for $u v$ y y $U$ B V R I J H K GU GB1 GB GB2 GV1 GV GGWWWUWLWBWV bands (Least-Squares Method).

\begin{tabular}{lccccc}
\hline \hline Name & Source & Range $T_{\text {eff }}$ & Range $\log g$ & Phot. System & Kind of fitting \\
\hline Table 2 & PHOENIX & $5000 \mathrm{~K}-10000 \mathrm{~K}$ & $3.0-5.5$ & Strömgren-Johnson & Eq. (5) \\
Table 3 & PHOENIX & $5000 \mathrm{~K}-10000 \mathrm{~K}$ & $3.0-5.5$ & Geneva-Walraven & Eq. (5) \\
Table 4 & PHOENIX & $5000 \mathrm{~K}-10000 \mathrm{~K}$ & $3.0-5.5$ & Strömgren-Johnson & Eq. (8) \\
Table 5 & PHOENIX & $5000 \mathrm{~K}-10000 \mathrm{~K}$ & $3.0-5.5$ & Geneva-Walraven & Eq. (8) \\
Table 6 & PHOENIX & $5000 \mathrm{~K}-10000 \mathrm{~K}$ & $3.0-5.5$ & Strömgren-Johnson & Eq. (1) (quasi spherical) \\
Table 7 & PHOENIX & $5000 \mathrm{~K}-10000 \mathrm{~K}$ & $3.0-5.5$ & Geneva-Walraven & Eq. (1) (quasi spherical) \\
Table 8 & PHOENIX & $5000 \mathrm{~K}-10000 \mathrm{~K}$ & $3.0-5.5$ & Strömgren-Johnson & Eq. (2) (quasi spherical) \\
Table 9 & PHOENIX & $5000 \mathrm{~K}-10000 \mathrm{~K}$ & $3.0-5.5$ & Geneva-Walraven & Eq. (2) (quasi spherical) \\
Table 10 & PHOENIX & $5000 \mathrm{~K}-10000 \mathrm{~K}$ & $3.0-5.5$ & Strömgren-Johnson & Eq. (3) (quasi spherical) \\
Table 11 & PHOENIX & $5000 \mathrm{~K}-10000 \mathrm{~K}$ & $3.0-5.5$ & Geneva-Walraven & Eq. (3) (quasi spherical) \\
Table 12 & PHOENIX & $5000 \mathrm{~K}-10000 \mathrm{~K}$ & $3.0-5.5$ & Strömgren-Johnson & Eq. (4) (quasi spherical) \\
Table 13 & PHOENIX & $5000 \mathrm{~K}-10000 \mathrm{~K}$ & $3.0-5.5$ & Geneva-Walraven & Eq. (4) (quasi spherical) \\
Table 14 & PHOENIX & $5000 \mathrm{~K}-10000 \mathrm{~K}$ & $3.0-5.5$ & Strömgren-Johnson & Eq. (5) (quasi spherical) \\
Table 15 & PHOENIX & $5000 \mathrm{~K}-10000 \mathrm{~K}$ & $3.0-5.5$ & Geneva-Walraven & Eq. (5) (quasi spherical) \\
Table 16 & PHOENIX & $5000 \mathrm{~K}-10000 \mathrm{~K}$ & $3.0-5.5$ & Strömgren-Johnson & Eq. (8) (quasi spherical) \\
Table 17 & PHOENIX & $5000 \mathrm{~K}-10000 \mathrm{~K}$ & $3.0-5.5$ & Geneva-Walraven & Eq. (8) (quasi spherical) \\
\hline
\end{tabular}
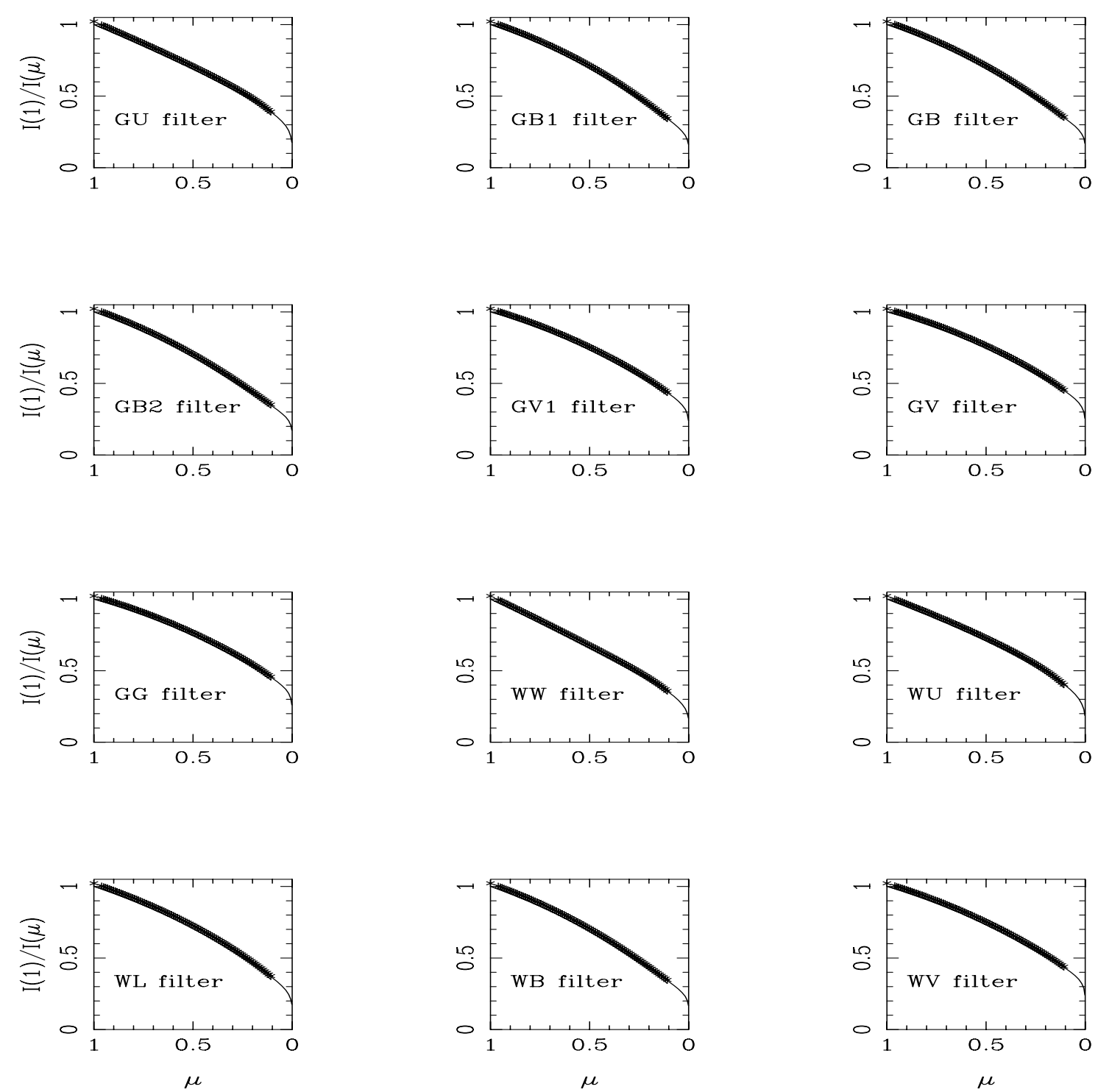

Fig. 6. The integrated and adjusted specific intensities for the model [7000, 4.0] (case "quasi" spherical, Geneva and Walraven systems). Dashed lines denote the actual integrated-intensities, asterisks the fits using Eq. (5) and full lines represent a plane-parallel model with the same effective temperature and surface gravity. 
This work was supported in part by NSF grants AST-9720704 and AST-0086246, NASA grants NAG5-8425, NAG5-9222, as well as NASA/JPL grant 961582 to the University of Georgia. This work was supported in part by the Pôle Scientifique de Modélisation Numérique at ENS-Lyon. Some of the calculations presented in this paper were performed on the IBM pSeries 690 of the Norddeutscher Verbund für Hoch- und Höchstleistungsrechnen (HLRN), on the IBM SP "seaborg" of the NERSC, with support from the DoE, and on the IBM SP "Blue Horizon" of the San Diego Supercomputer Center (SDSC), with support from the National Science Foundation. We thank all these institutions for a generous allocation of computer time.

\section{References}

Claret, A. 1998, A\&A, 335, 653

Claret, A. 2000, A\&A, 363, 1081

Claret, A. 2003, A\&A, 401, 657

Hauschildt, P. H., Allard, F., \& Baron, E. 1999, ApJ, 512, 377

Hauschildt, P. H., Allard, F., Ferguson, J,. Baron, E., \& Alexander, D. R. 1999, ApJ, 525, 871

Hauschildt, P. H., \& Baron, E. 1999, J. Comput. Appl. Math., 102, 41 Kurucz, R. 1993, Series of CDROMs

Orosz, J. A., \& Hauschildt, P. H. 2000, A\&A, 364, 265 